\title{
LIÇÕES APRENDIDAS NA IMPLEMENTAÇÃO DE ERP NA MODALIDADE SAAS: NA PERSPECTIVA DA EQUIPE DE PROJETO MULTILOCALIZADA E STAKEHOLDERS
}

\author{
LESSONS LEARNED IN ERP IMPLEMENTATION IN SAAS MODE: FROM THE \\ PERSPECTIVE OF THE MULTI-SITE PROJECT TEAM AND STAKEHOLDERS
}

\author{
Roberto Carlos Castro de Ponte \\ Mestre em Administração \\ Universidade Nove de Julho - UNINOVE. \\ São Paulo, SP - Brasil. \\ roberto_deponte@hotmail.com \\ Andressa Carolina Pessoa Lopes \\ Mestre em Administração \\ Universidade Nove de Julho - UNINOVE. \\ São Paulo, SP - Brasil. \\ andressacplopes@hotmail.com \\ Isabel Cristina Scafuto \\ Doutora em Administração \\ Universidade Nove de Julho - UNINOVE. \\ São Paulo, SP - Brasil. \\ isabelscafuto@gmail.com
}

\begin{abstract}
Resumo: $\mathrm{O}$ crescente aumento de banda de Internet e as rápidas mudanças nas necessidades de negócio, está levando as organizações a adotarem infraestruturas de sistemas de informação que sejam econômicas e flexíveis. O objetivo deste relato é apresentar na perspectiva da equipe de projeto multilocalizada e stakeholders, um modelo que foi elaborado por meio de lições aprendidas, que minimizará os desafios na implementação de Enterprise Resource Planning (ERP) em nuvem, na modalidade SaaS - Software as a Service. Para alcançar o objetivo proposto, os dados foram coletados da documentação do projeto, além da participação direta de um dos autores. Os resultados apresentam um modelo de implementação do ERP com soluções práticas para mitigarem os desafios de: mudança de processos na implementação do ERP; disponibilidade da equipe funcional para participação durante a validação e redesenho dos processos de negócio da organização; disponibilidade dos stakeholders para validação das atualizações constantes do produto; gerenciamento de equipes multilocalizadas. A contribuição do relato é, por meio das lições aprendidas, fornecer um modelo para as empresas que trabalham com implementação de sistemas.
\end{abstract}

Palavras-chave: ERP. SAAS. Equipe multilocalizada. Stakeholders. Lições aprendidas.

Abstract: The growing increase in Internet bandwidth and the rapid changes in business needs, is leading organizations to adopt information systems infrastructures that are economical and flexible. The purpose of this report is to present, from the perspective of the multilocalized project team and stakeholders, a model that was developed through lessons learned, which will mitigate the challenges in implementing cloud Enterprise Resource Planning (ERP), in the SaaS - Software as a Service model. To achieve the proposed objective, data were collected from the project documentation, in addition to the direct participation of one of the authors. The results present an ERP implementation model with practical solutions to mitigate the challenges of: changing processes in the implementation of ERP; functional team availability for participation during validation and redesign of the organization's business processes; stakeholders availability to validate software improvement updates; multi-localized teams management. The report contribution is, through lessons learned, to provide a model for companies that work with systems implementation.

Keywords: ERP. SAAS. Multilocation team. Stakeholders. Lessons learned.

\section{Cite como}

American Psychological Association (APA)

Ponte, R. C. C., Lopes, A. C. P., \& Scafuto, I. C. (2021, jan./jun.). Lições aprendidas na implementação de ERP na modalidade SAAS: na perspectiva da equipe de projeto multilocalizada e stakeholders. Revista Inovação, Projetos e Tecnologias - IPTEC, São Paulo, 9(1), 32-44. https://doi.org/10.5585/iptec.v9i1.18698. 


\section{Introdução}

As companhias estão transformando radicalmente suas estratégias referente à tecnologia da informação através da compra de software pré-embalados em vez de trabalharem no desenvolvimento de sistemas (Holland \& Luz, 1999; Mital, Pani, \& Ramesh, 2014). Consequentemente, sistemas de Enterprise Resource Planning Systems (ERP) são considerados uma relevante inovação empresarial e sua implementação visa beneficiar empresas de diversos portes (Sommerville, 2011). O software como um modelo de serviço Software as a Service (SaaS) está diligentemente se transformando em uma maneira rentável de entregar aplicações de negócios (Seethamraju, 2014).

O ERP é um conjunto de software empacotado com soluções abrangentes, que buscam integrar os processos e funções de uma empresa, com a finalidade de apresentar uma visão holística dos negócios a partir de uma única arquitetura de informação e Tecnologia da Informação (TI). Muitas organizações de grande porte em todo o mundo utilizam o ERP, cada vez mais pequenas e médias empresas (PMEs) também estão identificando a necessidade competitiva na adoção. Embora a ampla e estreita integração do ERP somente tenha se tornado disponível nos últimos anos, o ERP tem um 'pedigree' em software de aplicativos grandes e empacotados que têm sido amplamente utilizado desde a década de 1970 (Klaus, Rosemann, \& Gable, 2000).

Com o crescente uso dos ERPs, os riscos na sua implementação também estão surgindo (Benlian \& Hess, 2011). As empresas contam cada vez mais com equipes multilocalizadas. Isso ocorre porque a empresa contratada realiza remotamente o auxílio e suporte à empresa cliente (Bjorn \& Ngwenyama, 2009). Organizações globalmente distribuídas normalmente trazem pessoas de diferentes culturas, línguas e de locais heterogêneos para atuar em projetos específicos, criando os riscos. Estas equipes são denominadas equipe multilocalizadas ou virtuais, que colaboram para um objetivo compartilhado usando uma combinação de tecnologias de informação e comunicação para realização de tarefas (Bjorn \& Ngwenyama, 2009).

Esses projetos envolvem muitas pessoas, por isso, considerar quais os benefícios são aguardados com o projeto e o que de fato acatará as expectativas dos stakeholders é importante para as organizações na implementação do ERP (Keeys \& Huemann, 2017). Além disso, as empresas devem contar com as lições aprendidas para melhorarem cada vez mais os seus processos de implementação, propiciando aos stakeholders uma melhor experiência (Jugdev, 2012). 
Diante do exposto, este relato técnico apresenta os principais problemas e suas soluções, por meio de um modelo elaborado com lições aprendidas, para a implementação do sistema ERP na modalidade SaaS, conduzido por uma empresa multinacional de tecnologia dos Estados Unidos. A implementação foi realizada em uma companhia de médio porte de gestão de risco de saúde, que presta serviços ambulatoriais no Brasil para a população de baixa renda que, normalmente, não possui plano de saúde.

\section{Fundamentação teórica}

A fim de fundamentar este relato técnico, apresentamos uma revisão de conceitos sobre os assuntos discorridos, procurando expor conhecimentos fundamentais do desenvolvimento do trabalho. Os principais tópicos deste relato técnico são: ERP em SaaS; Stakeholder, Equipes Multilocalizadas e Lições Aprendidas.

\section{$2.1 E R P$}

Os ERPs substituem os diferentes sistemas de transações de uma empresa por um sistema integrado que incorpora interdependências entre os processos funcionais da organização (Kirmizi \& Kocaoglu, 2021). Porém, a implementação desses sistemas é demasiadamente onerosa. Conforme estudos de Ross e Vitale (2000), existem seis motivos comuns aos quais as empresas adotam o ERP e suas implementações, sendo estes: 1) necessidade de uma plataforma comum; 2) melhoria de processos; 3) visibilidade de dados; 4) redução de custos operacionais; 5) maior capacidade de resposta do cliente e 6) melhoria na tomada de decisões estratégicas. Essas razões mostraram-se inter-relacionadas, pois a plataforma de sistemas permitiu novos recursos que deveriam gerar importantes resultados de desempenho.

A computação em nuvem é uma tecnologia inovadora que continuará a liberar novas inovações e trazer novas eficiências e vantagens aos negócios (Ross \& Vitale, 2000). Ela elimina a infraestrutura e as despesas de capital como uma barreira à entrada e permite que as empresas de startups aumentem a escala de maneira barata e rápida. Por outro lado, as grandes corporações enfrentam limitações no uso da nuvem para aplicativos de alto desempenho e de missão crítica, como o ERP (Hofmann \& Woods, 2010). Cloud Computing é definido como um modelo para permitir acesso de rede onipresente, conveniente e sob demanda a um conjunto compartilhado de recursos de computação configuráveis, como redes, servidores, armazenamento, aplicativos e serviços, que podem ser rapidamente provisionados e liberados 
com o mínimo esforço de gerenciamento ou interação com provedores de serviço (Hsu, Ray, \& Hsieh, 2014).

Os aplicativos SaaS fornecidos por meio da nuvem fornecem uma opção de baixo capital e implantação rápida. Dependendo do aplicativo, é essencial integrá-lo a aplicativos tradicionais que podem residir em uma nuvem separada ou em uma tecnologia tradicional. O padrão de interoperabilidade é um facilitador ou uma barreira, permite a manutenção da integridade, consistência das informações e processos de uma empresa, de acordo com o estudo de Avram (2014).

Ainda sob a ótica de Avram (2014), computação em nuvem representa uma convergência de duas grandes tendências em tecnologia da informação: (a) eficiência de TI, em que o poder dos computadores modernos é utilizado de forma mais eficiente através de recursos de hardware e software altamente escaláveis e (b) agilidade nos negócios, na qual a TI pode ser usada como ferramenta competitiva por meio de implantação rápida, processamento em lote paralelo, uso de análise de negócios com uso intensivo de computação e aplicativos interativos móveis que respondem.

\subsection{Stakeholders}

Em Turner e Zolin (2012), são propostos oito tipos diferentes de partes interessadas: investidor ou proprietário; executivo do projeto ou patrocinador; consumidores, operadores/usuários; gerente e time do projeto; fornecedores principais; outros fornecedores e público, sendo que cada um dos oito tipos têm diferentes interesses nas saídas, resultados e impactos do projeto, além de maneiras diferentes de julgar o seu sucesso. Sendo assim, considerar quais os benefícios são aguardados com o projeto e o que de fato acatará as expectativas dos stakeholders é importante para as organizações (Keeys \& Huemann, 2017).

Com base em Marston et al. (2011), em uma configuração de computação tradicional, os stakeholders são os provedores e consumidores: onde os consumidores usam, mantêm e atualizam os sistemas, enquanto os provedores lidam com a venda, instalação, licenciamento, consultoria e manutenção da tecnologia envolvida. Entretanto, na adoção do SaaS, os stakeholders incluem não somente os provedores e consumidores do serviço, mas também em função do modelo de entrega do serviço, os técnicos e arquitetos precisam entender o impacto da localização da infraestrutura dos provedores de serviços (Marston et al., 2011). 


\subsection{Equipes multilocalizadas}

Daim et al. (2012) argumentam que equipes gerenciadas por matrizes virtuais com membros geograficamente dispersos, estão se tornando cada vez mais comuns no setor de tecnologia. Essas equipes, chamadas de equipes virtuais globais, geralmente são descritas como grupos de trabalho com comunicação eletrônica diversamente dispersos geograficamente. Eles rapidamente se formam, mudam e se dissolvem devido às mudanças dinâmicas no mercado (Daim et al., 2012).

A maioria das equipes virtuais têm membros da equipe espalhados entre vários projetos com prioridades concorrentes (Daim et al. 2012). O colapso da comunicação pode causar estragos em um projeto, pois os membros da equipe lutam para se comunicar e trabalhar de forma eficaz entre si. Como resultado, os riscos de entrega de projetos com equipes distribuídas tendem a ser maiores quando comparados a equipes co-localizadas (Daim et al., 2012). Neste sentido, gerir uma equipe multilocalizada implica em ter alguns cuidados com a integração, pois são influenciados por culturas diferentes (Arditi, Nayak, \& Damci, 2017).

A tecnologia móvel, a globalização e a internet, apoiadas pela disponibilidade de largura de banda, estão reduzindo a necessidade de interação humana presencial. A comunicação por meio de computador, desempenha um papel crescente na vida das pessoas e pode transformar a equipe virtual de uma fonte inovadora de vantagem competitiva para um modelo organizacional de projeto dominante. As tecnologias, como groupware, videoconferência, telefones celulares e internet, dão suporte ao trabalho das equipes multilocalizadas (Weimann et al., 2013).

\subsection{Lições aprendidas}

As lições aprendidas são um processo que tem como objetivo principal captar os resultados e experiências de sucessos e fracassos, aprendendo com eles e os incorporando na organização para que seja usado futuramente (McClory et al., 2017). A dispersão e o aproveitamento das lições aprendidas são fundamentais aos projetos para que as organizações possam alcançar o sucesso (Duffield \& Whitty, 2016), devendo ser incorporadas pela organização.

As pessoas acabam aprendendo mais com os fracassos do que com as vitórias. Mas, as falhas acabam contendo informações valiosas para serem usadas, mesmo que as organizações muitas vezes tenham dificuldades em aprender (McClory, et al., 2017). Nos projetos, por sua natureza provisória, já que possuem um início, um meio e um fim já determinados, as 
organizações acabam interrompendo o fluxo de conhecimento, criando uma barreira para a aprendizagem (McClory et al., 2017). Apesar disso, na literatura é possível encontrar que o sucesso do processo de lições aprendidas depende muito de uma organização com uma cultura para a aprendizagem (Duffield \& Whitty, 2016).

\section{Metodologia}

Conforme Protocolo para Elaboração de Relatos de Produção Técnica proposto por Biancolino, Kniess, Maccari e Rabechini Jr. (2012) este relato técnico consiste em uma experiência profissional, observada com rigor científico e metodológico. Desta forma, nesta seção serão detalhadas as atividades relevantes para elaboração do relato.

O desenvolvimento do relato é uma abordagem qualitativa com método de pesquisaação, vivenciada na função de gerente de projeto de implementação do ERP na modalidade SaaS. Devido a função exercida por um dos autores, a participação foi direta através de reuniões de negociação entre o patrocinador do projeto, usuários-chave e departamentos da empresa, por exemplo: financeiro, contas a pagar, contas a receber, controladoria, compras, faturamento, além de workshops técnicos e funcionais junto a supervisores de centros médicos, fornecedores de serviços e parceiros de implementação da solução.

Conforme descrito na Figura 1, observa-se o passo a passo ao qual se deu o levantamento de dados para o presente relato:

Figura 1 - Processo de coleta de dados
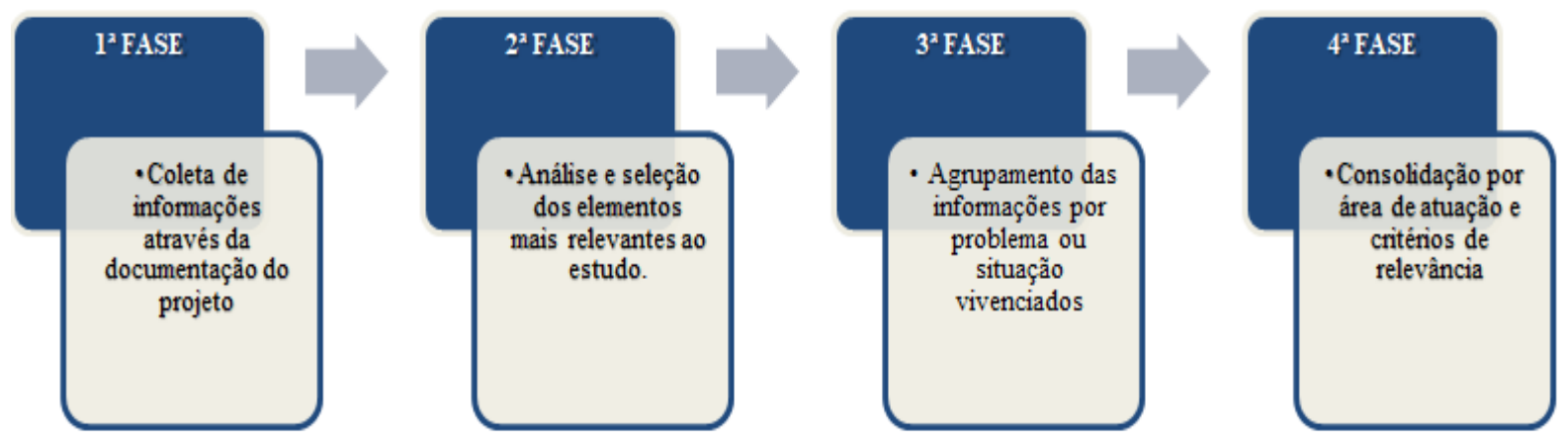

Fonte: Elaborado pelos autores (2020).

A primeira etapa do trabalho ocorreu pela coleta de informações por meio da documentação do projeto como minutas de reuniões, documentação do produto, e-mails e anotações referente aos problemas, alternativas de soluções, impactos e respostas a riscos e planos de ação. Com base nas informações coletadas sobre os problemas em questão, o processo 
de coleta prosseguiu, com a análise e seleção dos elementos mais relevantes ao estudo, em alguns casos requerendo informações secundárias originadas da equipe do projeto ou mesmo stakeholders. Todos os dados coletados foram agrupados em uma planilha de Excel para facilitar a sua análise.

Após a seleção das informações relevantes, a organização e estruturação das informações obtidas foram iniciadas, tomando como base o agrupamento das informações por problema ou situação vivenciados durante o ciclo de vida do projeto. O agrupamento das informações foi feito com os seguintes elementos: situação ou problema; ambiente impactado e nível de impacto; possíveis alternativas técnicas e/ou funcionais analisadas; abordagens junto aos stakeholders; e planos de ação adotados. Posteriormente, as informações foram consolidadas por área de atuação e critérios de relevância.

\section{Contexto do projeto}

Este relato apresenta lições aprendidas na implementação de ERP na modalidade SaaS, conduzido por uma empresa multinacional de tecnologia dos Estados Unidos. A implementação do sistema ocorreu em uma companhia de médio porte de gestão de risco de saúde que presta serviços ambulatoriais no Brasil para a população de baixa renda que, normalmente, não possui plano de saúde.

O ERP na modalidade SaaS, tem como característica principal os processos funcionais estabilizados e padronizados globalmente, ou seja, o motor principal do sistema é único para qualquer localidade do mundo. A sua funcionalidade de multi-linguagem, em caráter de exceção e os desenvolvimentos de customização, é desenvolvida e implementada em outro ambiente chamado de PaaS (Platform as a Service).

Os desafios são decorrentes das vantagens que o produto ERP, na modalidade SaaS, propõe na perspetiva dos stakeholders: o primeiro desafio na perspectiva dos stakeholders internos e externos, equipes funcionais, fornecedores e clientes, consiste nas mudanças de processos que atualmente estão em operação. Conforme Seethamraju (2014), as empresas que possuem processos, sistemas e funções mais estabelecidos, podem relutar em mudar. A rotina da organização é formada por atividades e procedimentos que formam os processos, que por sua vez, consolidam a cultura operacional da organização, sendo assim, o desafio consiste da ruptura desta cadeia atualmente consolidada e operacional.

O segundo desafio é relativo à disponibilidade da equipe funcional para a participação durante a validação e redesenho dos processos de negócio da organização. As organizações 
estão acostumadas a implementar sistemas no modelo on-premises, em que o sistema é adequado ou customizado para atender aos processos de negócio já definidos pela organização. Neste modelo, o envolvimento dos usuários finais na implementação do produto se concentra nos testes integrados e homologação do sistema. Porém, no modelo de entrega SaaS, os processos organizacionais serão reavalidados e algumas vezes redefinidos para alinhamento com os processos padrão do produto globalizado.

Assim, como o segundo, o terceiro desafio é referente à disponibilidade dos stakeholders. Neste caso, proveniente do esforço para validação das atualizações constantes do produto, normalmente quatro por ano. Estas atualizações são resultado de correções do produto, bugs de segurança e evolução do produto. A priorização das melhorias do produto é feita através de solicitações dos clientes, por meio de fóruns específicos, e roadmap do produto, significa dizer que as melhorias estão alinhadas com os desejos da maioria dos clientes que opinam sobre a sua evolução.

Por conta da padronização de ambiente e execução feita pela equipe multilocalizada do fornecedor do produto, o tempo de indisponibilidade do ambiente é menor que a manutenção em ambiente on-premisses e sua execução pode ser feita no melhor momento para o cliente independentemente do fuso horário. As atualizações ocorrem, primeiramente, no ambiente de teste e após duas semanas ocorre no ambiente de desenvolvimento e produção. Durante as duas semanas de defasagem entre atualização no ambiente de teste, desenvolvimento e produção, é esperado que os stakeholders validem os principais processos da organização e integrações com demais sistemas, para garantir que os processos produtivos e essenciais não serão afetados pela mudança. O desafio consiste no esforço e na disponibilidade dos stakeholders na validação do sistema após cada atualização do produto.

O quarto desafio é o gerenciamento de equipes multilocalizadas durante o projeto de implementação e sustentação do produto. Em função da especialização do suporte e da necessidade de atendimento vinte e quatro dias por semana, durante sete dias da semana em fusos horários distintos, estas equipes estão distribuídas pelos cinco continentes. Apesar das equipes estarem distantes umas das outras, seguindo processos, línguas e culturas distintas, trabalham juntas com objetivo comum na implementação do projeto ou resolução de um problema durante o ciclo de contratação do produto ERP em modalidade SaaS. 


\section{Tipos de intervenção e mecanismos adotados}

Neste tópico do relato são reportados os resultados e intervenções executadas para os desafios encontrados durante a implementação do ERP em modalidade SaaS, conforme descrito na Figura 2. Para o primeiro desafio que é a aceitação por parte dos stakeholders das mudanças sobre os processos atualmente em operação, a equipe do projeto elaborou e executou workshops. No material apresentado, são mostrados os fluxos de trabalho detalhando processos nativos da ferramenta ERP em modalidade SaaS, assim como parametrizações disponíveis para atender aos diversos requisitos dos clientes, utilizando modelos e exemplos na mesma área de atuação da organização em questão.

Figura 2 - Intervenções sobre desafios enfrentados

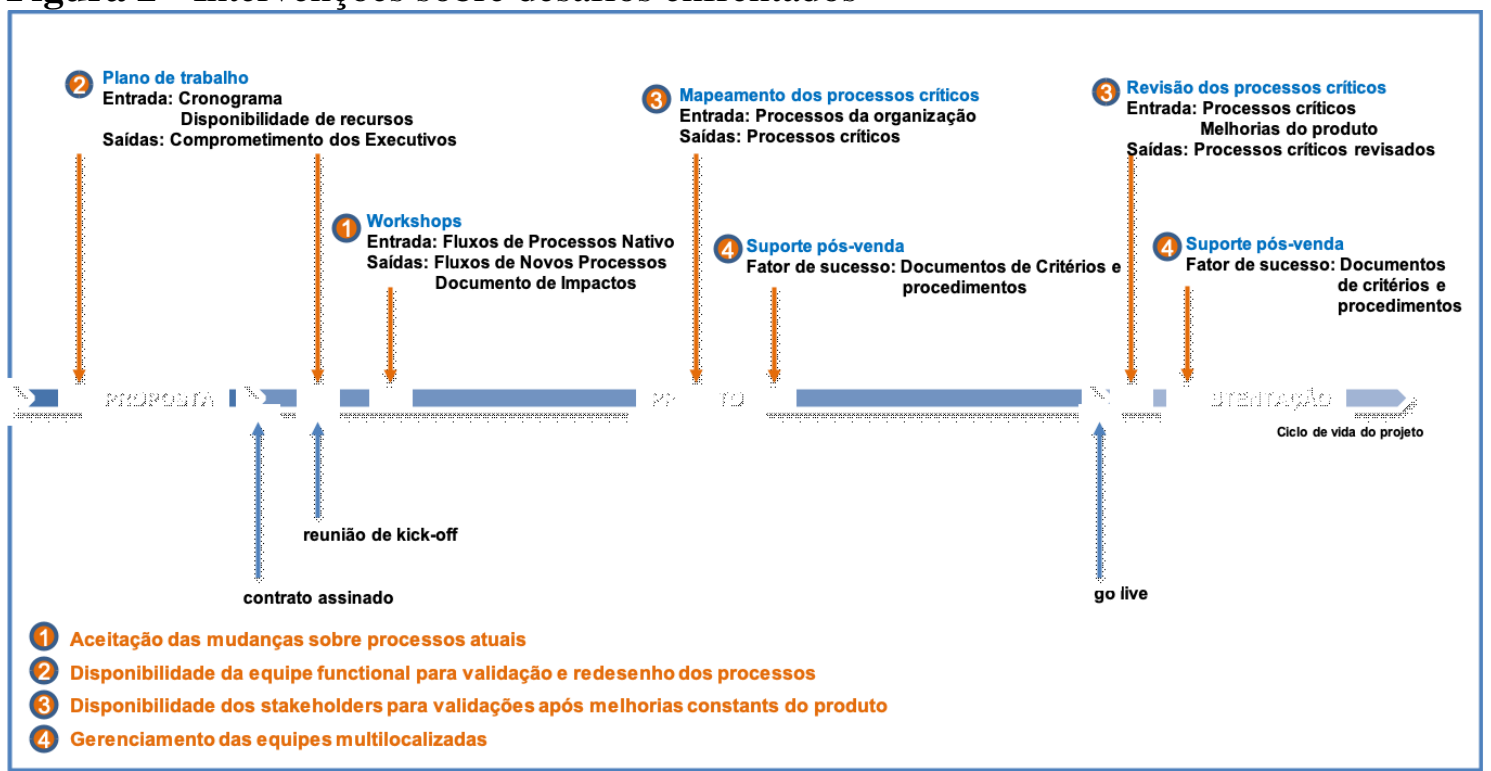

Fonte: Elaborado pelos autores (2020).

O objetivo é sensibilizar os stakeholders para os processos estabilizados e operacionais em organizações do mesmo seguimento. As saídas do plano de ação são fluxos de trabalho, detalhando os novos processos, documento de impactos funcionais e organizações durante e após a implementação dos novos processos. Essa solução apresentada é proveniente de lições aprendidas que envolveram problemas com mudanças enfrentadas pelos stakeholders.

No plano de ação referente ao segundo desafio de disponibilidade da equipe funcional para participação de validações e redesenho dos processos de negócio da organização, a equipe do projeto elaborou documentos de utilização de recursos alinhado ao cronograma do projeto e fatores críticos de sucesso do projeto com base em lições aprendidas. Estes documentos são apresentados em tempo de venda, anexado a proposta e no início do projeto durante a reunião de kick-off. As saídas deste plano de ação é o comprometimento dos executivos da organização 
em relação à disponibilidade dos stakeholders durante o processo de validação e redefinição dos processos organizacionais.

Para mitigar o terceiro desafio, o plano de ação iniciou durante a implementação do projeto. Neste momento os stakeholders efetuaram o mapeamento dos processos críticos para garantir a continuidade da operacionalização da organização, considerando o fluxo de trabalho do início ao fim do processo, envolvendo os sistemas integrados ao ERP. Posteriormente, os stakeholders definiram os principais cenários, independentemente do sistema, com as devidas informações de entrada e resultados esperados. Para que os testes pudessem ser realizados, uma massa de teste foi elaborada para atender a cada cenário.

Antes da alteração ser liberada aos clientes, o fornecedor do produto disponibiliza a documentação com as correções aplicadas, bugs corrigidos e melhorias. A partir desta documentação, os stakeholders devem avaliar os impactos no ambiente produtivo e atualizar os planos de testes. Com base nos cenários e massa de teste, foi acordado com a equipe multilocalizada de sustentação ao ambiente, contratada pela organização, para ser responsável pelos testes e geração de evidências para os stakeholders validarem o resultado real com o esperado para aprovar a implementação da alteração no ambiente produtivo. Desta maneira, o esforço dos stakeholders internos se concentra nas análises de impacto das mudanças frente aos processos críticos e validação das evidências geradas pela equipe de sustentação.

O gerenciamento da equipe multilocalizada, que é quarto desafio elencado neste relato, teve como mecanismo a nomeação de um gerente pós-venda para acompanhar os diferentes processos entre as equipes multilocalizadas. A função deste gerente pós-venda é suportar o gerente de projeto durante a implementação do ERP e após a implementação, assistir aos stakeholders da organização em processos de escalonamento e interação com as equipes multilocalizadas. O fator crítico de sucesso para este mecanismo é a definição e acompanhamento de regras básicas conhecidas como ground rules, como definição de critérios e procedimentos de escalação, documentação requerida por processo, meio de comunicação formal e informal, além de procedimentos para comunicação eficiente. Todas as soluções para a implantação de um novo sistema ERP que foram expostas, são soluções advindas de lições aprendidas ao longo do tempo e foram cruciais para o sucesso dos projetos e a vantagem competitiva da empresa relatada. 


\section{Contribuição tecnológica/social}

O objetivo do trabalho foi alcançado com a apresentação de lições aprendidas na implementação de ERP na modalidade SaaS, conduzido por uma empresa multinacional de tecnologia dos Estados Unidos. A implementação foi realizada em uma companhia de médio porte de gestão de risco de saúde que presta serviços ambulatoriais no Brasil para a população de baixa renda que, normalmente, não possui plano de saúde. Com base nos desafios encontrados durante o projeto, na perspectiva dos stakeholders com equipe de suporte multilocalizada, foram apresentadas interações a fim de abrandar os principais pontos de atenção na implementação do ERP em SaaS. O modelo apresentado foi elaborado com base em lições aprendidas da empresa relatada ao longo do tempo.

A principal contribuição deste relato técnico é apresentar uma visão realista dos desafios na perspectiva dos stakeholders, com equipe de suporte multilocalizada para os projetos subsequentes de implementação de Enterprise Resource Planning Systems (ERP) em modalidade SaaS (Software as a Service). Os profissionais de gerenciamento de projetos poderão encontrar neste relato, as lições aprendidas com aplicação prática de interações pontuais para mitigar os desafios enfrentados durante a situação apresentada. Enquanto, para os usuários finais, este relato contribui no esclarecimento de que apesar da recente tecnologia e complexidade de impostos da região, a implementação de ERP em SaaS é uma realidade no Brasil, com desafios e seus respectivos planos de ação elencados.

Uma das limitações do relato é que o seu desenvolvimento ficou restrito a perspectiva dos stakeholders. Como pesquisa futura, espera-se que este estudo seja parte de um trabalho mais amplo, sendo recomendado, então, a captura das lições aprendidas a partir da maturidade das equipes e maior complexidade dos ambientes envolvidos. Com isso, pode-se atualizar o modelo apresentado.

As empresas que trabalham com implementação de sistemas poderão adaptar o modelo aqui apresentado (Figura 2) para a sua própria realidade. Cada desafio que foi detalhado neste relato e que foi apresentada uma solução com as lições aprendidas, poderá facilitar para outras empresas que poderão pular essa longa etapa de aprendizado mostrado nesse estudo. 


\section{Referências}

Arditi, D., Nayak, S., \& Damci, A. (2017). Effect of organizational culture on delay in construction. International Journal of Project Management, 35, 136-147.

Avram, M. G. (2014). Advantages and challenges of adopting cloud computing from an enterprise perspective. Procedia Technology Systems, 12(1), 529-534.

Benlian A. \& Hess, T., (2011). Opportunities and risks of software-as-a-service: Findings from a survey of IT executives. Decision Support Systems, 52(1), 232-246.

Biancolino, C. A., Kniess, C. T., Maccari, E. A., \& Rabechini, R., Jr. (2012). Protocolo para elaboração de relatos de produção técnica. Revista de Gestão e Projetos - GeP, 3(2), 294 307.

Bjorn, P., \& Ngwenyama, O. (2009). Virtual team collaboration: building shared meaning, resolving breakdowns and creating translucence. Information Systems Journal, 19(3), 227253.

Daim, T. U., Ha, A., Reutiman, S., Hughes, B, Pathak, U., Bynum, W. \& Bhatla, A. (2012). Exploring the communication breakdown in global virtual teams. International Journal of Project Management, 30(2), 199-212.

Duffield, S. M. \& Whitty, S. J. (2016). Application of the Systemic Lessons Learned Knowledge model for Organisational Learning through Projects. International Journal of Project Management, 34(7), 1280-1293.

Hofmann, P. \& Woods, D. (2010). Cloud Computing: The Limits of Public Clouds for Business Applications. IEEE Internet Computing, 14(6), 90-93.

Holland, C. P., \& Light, B. (1999). A Critical Success Factors Model For ERP Implementation. IEEE Software, 16, 30-36.

Hsu, P. F., Ray, S. \& Hsieh, Y. L. (2014). Examining cloud computing adoption intention, pricing mechanism, and deployment model. International Journal of Information Management, 34(4), 474-488.

Jugdev, K. (2012). Learning from lessons learned: Project management research program. American Journal of Economics and Business Administration, 4(1), 13.

Keeys, L. A., \& Huemann, M. (2017). Project benefits co-creation: Shaping sustainable development benefits. International Journal of Project Management, 35(6), 1196-1212.

Kirmizi, M. \& Kocaoglu, B. (2021). The influencing factors of enterprise resource planning (ERP) readiness stage on enterprise resource planning project success: a project manager's perspective. Kybernetes, ahead-of-print.

Klaus, H., Rosemann, M. \& Gable, G. G. (2000). What is ERP?. Information Systems Frontiers, 2(2), 141-162. 
Marston, S., Li, Z., Bandyopadhyay, S., Zhang, J., \& Ghalsasi, A., (2011). Cloud computing The business perspective. Decision Support Systems, 51(1), 176-189.

McClory, S., Read, M. \& Labib, A. (2017). Conceptualising the lessons-learned process in project management: Towards a triple-loop learning framework. International Journal of Project Management, 35 (7), 1322-1335.

Mital, M., Pani, A. K., \& Ramesh, R., (2014). Determinants of choice of semantic web based Software as a Service: An integrative framework in the context of e-procurement and ERP. Computers in Industry, 65(5), 821-827.

Ross, J. W. \& Vitale, M. R., (2000). The ERP Revolution: Surviving vs. Thriving. Information Systems Frontiers, 2(2), 233-241.

Seethamraju, R. (2014). Adoption of Software as a Service (SaaS) Enterprise Resource Planning (ERP) Systems in Small and Medium Sized Enterprises (SMEs). Information Systems Frontiers, 17(3), 475-492.

Sommerville, I. (2011). Engenharia de Software. 9 ed, São Paulo: Pearson Prentice Hall.

Turner, R. \& Zolin, R. (2012). Forecasting success on large projects: developing reliable scales to predict multiple perspectives by multiple stakeholders over multiple time frames. Project Management Journal, 43 (5), 87-99.

Weimann, P., Pollock, M., Scott, E. \& Brown, I. (2013). Enhancing Team Performance Through Tool Use: How Critical Technology-Related Issues Influence the Performance of Virtual Project Teams. IEEE Transactions on Professional Communication, 56(4), 332-353 